\title{
On $p$ th moment exponential stability of stochastic differential equations with Markovian switching and time-varying delay
}

\section{Enwen Zhu*, Xue Tian and Yueheng Wang}

"Correspondence:

engwenzhu@163.com School of Mathematics and

Computing Science, Changsha University of Science and Technology, Changsha, Hunan 410004, P.R. China

\begin{abstract}
In this paper we discuss the problem of pth moment exponential stability for general nonlinear stochastic differential equations with Markovian switching and time-varying delay. By using the Lyapunov function, the stochastic analysis technique and the generalized Halanay inequality, some novel sufficient conditions on pth moment exponential stability of stochastic differential equations with Markovian switching and time-varying delay are derived. The results obtained in this paper are completely new and modify and improve some known results. Moreover, two numerical examples are also provided to demonstrate the effectiveness and applicability of the theoretical results. The aim of this paper is to investigate $p$ th moment exponential stability of general nonlinear stochastic differential equations with Markovian switching and time-varying delay.
\end{abstract}

Keywords: pth moment exponential stability; time-varying delay; generalized Halanay inequality; Markovian switching

\section{Introduction}

In the last decades, the stability of stochastic differential equations has been extensively studied by many authors (see, e.g., [1-6]). Recently, the stability of stochastic differential equations with Markovian switching (or jumping) has received a lot of attention. For example, Ji and Chizeck [7] and Mariton [8] studied the stability of a jump linear equation. Basak et al. [9] discussed the stability of a semi-linear stochastic differential equation with Markovian switching. Mao [10] as well as Shaikhet [11] investigated the stability for nonlinear stochastic differential delay equations with Markovian switching. Especially, for a systematic and detailed review about stochastic differential equations with Markovian switching, Mao and Yuan [12] should be referred to.

Most of these papers are concerned with asymptotics in the probability or in mean square, while to the best of our knowledge, very few studies on $p$ th moment exponential stability of stochastic differential delay equations with Markovian switching. We should point out that [10], Theorem 4.1 investigated $p$ th moment exponential stability for general nonlinear stochastic differential equations with Markovian switching and constant delay, however, this theorem cannot apply to the case of stochastic differential equations with Markovian switching and variable time delay. We should also point out that [12], Theorem 7.23 discussed $p$ th moment exponential stability for a general nonlinear stochastic 
differential equation with Markovian switching and time-varying delay under the following assumptions: the delay functions are differentiable and the corresponding derivatives are simultaneously required to be not greater than 1 . Obviously these impose a very strict constraint on the model because time delays sometimes vary dramatically with time in real models $[13,14]$.

Motivated by the above discussions, the purpose of this paper is to study $p$ th moment exponential stability of a general nonlinear stochastic differential equation with Markovian switching and time-varying delay. By virtue of the generalized Halanay inequality and using stochastic analysis techniques, we establish a set of novel sufficient criteria for $p$ th moment exponential stability. Finally, the effectiveness of the proposed methods is illustrated by examples.

\section{Model description, notations, and assumptions}

Notations The notations are quite standard. Throughout this paper, $\mathbb{R}^{n}$ and $\mathbb{R}^{n \times m}$ denote, respectively, the $n$ dimensional Euclidean space and the set of all $n \times m$ real matrices. The superscript $T$ denotes the transpose. $I$ is the identity matrix with compatible dimension. For a fixed $\tau \in[0, \infty)$ denoting the delay or time-lag, let $\mathcal{C}:=C\left([-\tau, 0] ; \mathbb{R}^{n}\right)$ be the family of continuous functions $\zeta:[-\tau, 0] \mapsto \mathbb{R}^{n}$ with the norm $\|\zeta\|:=\sup _{-\tau \leq \theta \leq 0}|\zeta(\theta)|$, where $|\cdot|$ is the Euclidean norm in $\mathbb{R}^{n}$. If $A$ is a matrix, we denote by $\|A\|$ its operator norm, i.e., $\|A\|=$ $\sup \{|A x|:|x|=1\}=\sqrt{\lambda_{\max }\left(A^{T} A\right)}$ where $\lambda_{\max }(\cdot)$ (respectively, $\left.\lambda_{\min }(\cdot)\right)$ means the largest (respectively, smallest) eigenvalue of $A$. Moreover, let $\left(\Omega, \mathcal{F},\left\{\mathcal{F}_{t}\right\}_{t \geq 0}, \mathbb{P}\right)$ be a complete probability space with a filtration $\left\{\mathcal{F}_{t}\right\}_{t \geq 0}$ (i.e., it is right-continuous and $\mathcal{F}_{0}$ contains all $\mathbb{P}$-null sets). Denote by $C_{\mathcal{F}_{0}}^{b}\left([-\tau, 0] ; \mathbb{R}^{n}\right)$ the family of all bounded, $\mathcal{F}_{0}$-measurable, $C\left([-\tau, 0] ; \mathbb{R}^{n}\right)$ valued random variables. For $p>2$ and $\tau>0$, denote by $L_{\mathcal{F}_{0}}^{p}\left([-\tau, 0] ; \mathbb{R}^{n}\right)$ the family of all $\mathcal{F}_{0}$-measurable $C\left([-\tau, 0] ; \mathbb{R}^{n}\right)$-valued random variables $\phi=\{\phi(\theta):-\tau \leq \theta \leq 0\}$ such that $\sup _{-\tau \leq \theta \leq 0} \mathbb{E}|\phi(\theta)|^{p}<\infty$, where $\mathbb{E}$ stands for the corresponding expectation operator with respect to the given probability measure $\mathbb{P} . \omega(t)=\left(\omega_{1}(t), \ldots, \omega_{m}(t)\right)^{T}$ is an $m$-dimensional Brownian motion defined on a complete probability space $(\Omega, \mathcal{F}, \mathbb{P})$ with a natural filtration $\left\{\mathcal{F}_{t}\right\}_{t \geq 0}$ (i.e., $\mathcal{F}_{t}=\sigma\{\omega(s): 0 \leq s \leq t\}$ and $\mathcal{F}_{0}$ contains all $\mathbb{P}$-null sets).

Let $\{r(t)\}_{t \geq 0}$ be a right-continuous irreducible homogeneous Markov chain defined on the probability space $(\Omega, \mathcal{F}, \mathbb{P})$ taking values in a finite state space $\mathbb{S}:=\{1,2, \ldots, N\}$ with generator $\Gamma:=\left(\gamma_{i j}\right)_{N \times N}$ given by

$$
\mathbb{P}\{r(t+\Delta)=j \mid r(t)=i\}= \begin{cases}\gamma_{i j} \Delta+o(\Delta) & \text { if } i \neq j \\ 1+\gamma_{i i} \Delta+o(\Delta) & \text { if } i=j\end{cases}
$$

where $\Delta>0, \lim _{\Delta \rightarrow 0} o(\Delta) / \Delta=0, \gamma_{i j} \geq 0$ is the transition rate from $i$ to $j$, if $i \neq j$, while $\gamma_{i i}=-\sum_{j \neq i} \gamma_{i j}$. We assume that the Markov chain $\{r(t)\}_{t \geq 0}$ is independent of the Brownian motion $\{\omega(t)\}_{t \geq 0}$.

Consider a stochastic differential equation with Markovian switching and time-varying delay of the form

$$
d x(t)=f(x(t), x(t-\tau(t)), t, r(t)) d t+g(x(t), x(t-\tau(t)), t, r(t)) d \omega(t)
$$

on $t \geq 0$, with initial conditions $r(0)=i_{0} \in \mathbb{S}$ and $x(s)=\xi(s) \in C_{\mathcal{F}_{0}}^{b}\left([-\tau, 0] ; \mathbb{R}^{n}\right)$ for $s \in$ $[-\tau, 0]$. Here $f: \mathbb{R}^{n} \times \mathbb{R}^{n} \times \mathbb{R}^{+} \times \mathbb{S} \rightarrow \mathbb{R}^{n}$ and $g: \mathbb{R}^{n} \times \mathbb{R}^{n} \times \mathbb{R}^{+} \times \mathbb{S} \rightarrow \mathbb{R}^{n \times m}$ are mea- 
surable mappings, $x(t)=\left(x_{1}(t), \ldots, x_{n}(t)\right)^{T}$ and $\tau(t):[0, \infty) \rightarrow[0, \tau]$ is a Borel measurable function.

In order to guarantee the existence and uniqueness of the solution to (2.1), we shall impose some assumptions:

(H1) Both $f$ and $g$ satisfy the local Lipschitz condition. That is, for each $k=1,2, \ldots$, there is an $h_{k}>0$ such that

$$
|f(x, y, t, i)-f(\bar{x}, \bar{y}, t, i)|+|g(x, y, t, i)-g(\bar{x}, \bar{y}, t, i)| \leq h_{k}(|x-\bar{x}|+|y-\bar{y}|)
$$

for all $t \geq 0, i \in \mathbb{S}$ and those $x, y, \bar{x}, \bar{y} \in \mathbb{R}^{n}$ with $|x| \vee|y| \vee|\bar{x}| \vee|\bar{y}| \leq k$.

(H2) Both $f$ and $g$ satisfy the linear growth condition, that is, there is an $h>0$ such that

$$
|f(x, y, t, i)+g(x, y, t, i)| \leq h(1+|x|+|y|)
$$

for all $x, y \in \mathbb{R}^{n}, t \geq 0$, and $i \in \mathbb{S}$.

(H3) $f(0,0, t, i)=0, g(0,0, t, i)=0$, where $i \in \mathbb{S}, t \in \mathbb{R}^{+}$.

Throughout this paper, we always assume that $f$ and $g$ satisfy the usual local Lipschitz condition and linear growth condition. It follows from [10] that, for any given initial data $r(0) \in \mathbb{S}$ and $x_{0}=\xi \in C_{\mathcal{F}_{0}}^{b}\left([-\tau, 0] ; \mathbb{R}^{n}\right)$, system (2.1) has a unique global solution on $t \in \mathbb{R}^{+}$ and we denote the solution by $x(t ; r(0), \xi)$. For notational simplicity, we write $x(t ; r(0), \xi)=$ $x(t)$.

Let $C^{2,1}\left(\mathbb{R}^{n} \times \mathbb{R}^{+} \times \mathbb{S} ; \mathbb{R}^{+}\right)$denote the family of all non-negative functions $V(x, t, i)$ on $\mathbb{R}^{n} \times \mathbb{R}^{+} \times \mathbb{S}$ which are twice continuously differentiable in $x$ and once differentiable in $t$. For $V \in C^{2,1}\left(\mathbb{R}^{n} \times \mathbb{R}^{+} \times \mathbb{S} ; \mathbb{R}^{+}\right)$, we define an operator from $\mathbb{R}^{n} \times \mathbb{R}^{n} \times \mathbb{R}^{+} \times \mathbb{S}$ to $\mathbb{R}$ as follows:

$$
\begin{aligned}
\mathscr{L} V(x, y, t, i)= & V_{t}(x, t, i)+V_{x}(x, t, i) f(x, y, t, i) \\
& +\frac{1}{2} \operatorname{trace}\left[g^{T}(x, y, t, i) V_{x x}(x, t, i) g(x, y, t, i)\right]+\sum_{j=1}^{N} \gamma_{i j} V(x, t, j),
\end{aligned}
$$

where

$$
\begin{aligned}
& V_{t}(x, t, i)=\frac{\partial V(x, t, i)}{\partial t}, \quad V_{x}(x, t, i)=\left(\frac{\partial V(x, t, i)}{\partial x_{1}}, \ldots, \frac{\partial V(x, t, i)}{\partial x_{n}}\right), \\
& V_{x x}(x, t, i)=\left(\frac{\partial^{2} V(x, t, i)}{\partial x_{i} \partial x_{j}}\right)_{n \times n} .
\end{aligned}
$$

Under Hypothesis (H3), system (2.1) admits a trivial solution $x\left(t ; i_{0}, 0\right)=0$ corresponding to the initial data $\xi=0$ and $r(0)=i_{0}$.

The following definitions will be used in the sequel.

Definition 1 The trivial point of system (2.1) is said to be $p$ th moment exponentially stable if there exist $\alpha>0$ and $\beta>0$ such that

$$
E|x(t ; \xi)|^{p} \leq \alpha e^{-\beta t} \sup _{-\tau \leq \theta \leq 0} E|\xi(\theta)|^{p}, \quad t \geq 0
$$

holds for any $\xi \in C_{\mathcal{F}_{0}}^{b}\left([-\tau, 0] ; \mathbb{R}^{n}\right)$. 
Definition 2 For a continuous function $h: \mathbb{R} \rightarrow \mathbb{R}$, the upper right Dini-derivative $d^{+} h$ of $h$ is defined as

$$
d^{+} h(t)=\limsup _{\delta \rightarrow 0^{+}} \frac{h(t+\delta)-h(t)}{\delta} .
$$

\section{Main results}

In this section, we first present a lemma and a proposition which help us prove our main results.

Lemma 1 (Hardy inequality, [15], Lemma 1) Assume that there exist constants $a_{k} \geq 0$, $p_{k} \geq 0(k=1,2, \ldots, m+1)$, then the following inequality holds:

$$
\left(\prod_{k=1}^{m+1} a_{k}^{p_{k}}\right)^{\frac{1}{s_{m+1}}} \leq\left(\sum_{k=1}^{m+1} p_{k} a_{k}^{r}\right)^{\frac{1}{r}} S_{m+1}^{\frac{-1}{r}},
$$

where $r>0$ and $S_{m+1}=\sum_{k=1}^{m+1} p_{k}$. In (3.1), if we let $p_{m+1}=1, r=S_{m+1}=\sum_{k=1}^{m} p_{k}+1$, we will get

$$
\left(\prod_{k=1}^{m} a_{k}^{p_{k}}\right) a_{m+1} \leq \frac{1}{r}\left(\sum_{k=1}^{m} p_{k} a_{k}^{r}\right)+\frac{1}{r} a_{m+1}^{r},
$$

if we let $p_{m+1}=2, r=S_{m+1}=\sum_{k=1}^{m} p_{k}+2$, we will get

$$
\left(\prod_{k=1}^{m} a_{k}^{p_{k}}\right) a_{m+1} \leq \frac{1}{r}\left(\sum_{k=1}^{m} p_{k} a_{k}^{r}\right)+\frac{2}{r} a_{m+1}^{r} .
$$

Proposition 1 Assume that there are positive continuous functions $\lambda_{1}(t, i), \lambda_{2}(t, i)(i \in \mathbb{S})$ such that $0<\lambda_{0} \leq \lambda_{1}(t, i)$ and $0<\lambda_{2}(t, i) \leq q \lambda_{1}(t, i)$ with $0 \leq q<1$ for all $t \in \mathbb{R}^{+}$, and $y(t, i)$ $(i \in \mathbb{S})$ is a non-negative continuous function on $[-\tau, \infty]$ and satisfies the following inequality:

$$
d^{+} y(t, i) \leq-\lambda_{1}(t, i) y(t, i)+\lambda_{2}(t, i) \bar{y}(t, i)
$$

on $t \in \mathbb{R}^{+}$, where $\bar{y}(t, i)=\sup _{t-\tau \leq s \leq t}\{y(s, r(s))\}, \tau \geq 0$ is a constant. Then for all $t \in \mathbb{R}^{+}$, we have

$$
y(t, i) \leq \tilde{y}(t, i) e^{-\lambda^{*} t},
$$

where $\tilde{y}(t, i)=\sup _{-\tau \leq t \leq 0}|y(t, r(t))|$, and $\lambda^{*}>0$ is defined as

$$
\lambda^{*}=\inf _{t \in \mathbb{R}^{+}}\left\{\lambda(t, i): \lambda(t, i)=\lambda_{1}(t, i)-\lambda_{2}(t, i) e^{\lambda(t, i) \tau}\right\}
$$

Proof Note that the result is trivial if $\tau=0$. In the following we assume that $\tau>0$. Denote

$$
H(\lambda)=\lambda-\lambda_{1}(t, i)+\lambda_{2}(t, i) e^{\lambda \tau} .
$$


By the condition $0<\lambda_{0} \leq \lambda_{1}(t, i), 0<\lambda_{2}(t, i) \leq q \lambda_{1}(t, i)$ for all $t \in \mathbb{R}^{+}$, we have $H(0)=$ $-\lambda_{1}(t, i)+\lambda_{2}(t, i) \leq-(1-q) \lambda_{1}(t, i) \leq(q-1) \lambda_{0}<0, \lim _{\lambda \rightarrow \infty} H(\lambda)=\infty$, and $H^{\prime}(\lambda)=1+$ $\lambda_{2}(t, i) \tau e^{\lambda \tau}>0$ for any given fixed $t \in \mathbb{R}^{+}$. So $H(\lambda)$ is a strictly monotone increasing function. Therefore for any $t \in \mathbb{R}^{+}$there is a unique positive $\lambda(t, i)$ such that $\lambda(t, i)-\lambda_{1}(t, i)+$ $\lambda_{2}(t, i) e^{\lambda(t, i) \tau}=0$. From the definition, one has $\lambda^{*} \geq 0$. We have to prove $\lambda^{*}>0$. Suppose this is not true. Fix $\tilde{q}$ satisfying $0 \leq q<\tilde{q}<1$ and choose $\epsilon<\min \left\{\left(\frac{1}{\tau}\right) \ln \left(\frac{1}{\tilde{q}}\right),\left(1-\frac{q}{\tilde{q}}\right) \lambda_{0}\right\}$. Then there is $t^{*} \geq 0$ such that $\tilde{\lambda}\left(t^{*}, i\right)<\epsilon$ and $\tilde{\lambda}\left(t^{*}, i\right)-\lambda_{1}\left(t^{*}, i\right)+\lambda_{2}\left(t^{*}, i\right) e^{\tilde{\lambda}\left(t^{*}, i\right) \tau}=0$. Now we have

$$
\begin{aligned}
0 & =\tilde{\lambda}\left(t^{*}, i\right)-\lambda_{1}\left(t^{*}, i\right)+\lambda_{2}\left(t^{*}, i\right) e^{\tilde{\lambda}\left(t^{*}, i\right) \tau} \\
& <\epsilon-\lambda_{1}\left(t^{*}, i\right)+\lambda_{2}\left(t^{*}, i\right) e^{\epsilon \tau} \\
& <\epsilon-\lambda_{1}\left(t^{*}, i\right)+\frac{1}{\tilde{q}} \lambda_{2}\left(t^{*}, i\right) \\
& \leq \epsilon-\lambda_{1}\left(t^{*}, i\right)+\frac{q}{\tilde{q}} \lambda_{1}\left(t^{*}, i\right) \\
& =\epsilon-\left(1-\frac{q}{\tilde{q}}\right) \lambda_{1}\left(t^{*}, i\right) \\
& \leq \epsilon-\left(1-\frac{q}{\tilde{q}}\right) \lambda_{0}<0,
\end{aligned}
$$

which is a contradiction.

For any given $k>1$, set $x(t, i)=k \tilde{y}(t, i) e^{-\lambda^{*} t}$ and $z(t, i)=x(t, i)-y(t, i)$. Suppose (3.5) fails. Then there exists a $k>1$ such that

$$
y(t, i)>k \tilde{y}(t, i) e^{-\lambda^{*}(t, i) t}
$$

for some $t \in \mathbb{R}^{+}$. Let $\zeta=\inf \{t \geq 0: x(t, i)-y(t, i) \leq 0\}$. Then we have for some $\zeta>\tau$ that

$$
z(\zeta)=x(\zeta)-y(\zeta)=0 \quad \text { and } \quad z^{\prime}(\zeta)=x^{\prime}(\zeta)-y^{\prime}(\zeta) \leq 0
$$

Hence

$$
\begin{aligned}
z^{\prime}(\zeta) & =x^{\prime}(\zeta)-y^{\prime}(\zeta) \\
& \geq-k \tilde{y}(t, i) \lambda^{*} e^{-\lambda^{*} \zeta}-\left[-\lambda_{1}(\zeta, i) y(\zeta, i)+\lambda_{2}(\zeta, i) \bar{y}(\zeta, i)\right] \\
& >-k \tilde{y}(t, i) \lambda^{*} e^{-\lambda^{*} \zeta}+k \tilde{y}(t, i) \lambda_{1}(\zeta, i) e^{-\lambda^{*} \zeta}-k \tilde{y}(t, i) \lambda_{2}(\zeta, i) e^{-\lambda^{*}(\zeta-\tau)} \\
& \geq k \tilde{y}(t, i) e^{-\lambda^{*} \zeta}\left[-\lambda^{*}+\lambda_{1}(\zeta, i)-\lambda_{2}(\zeta, i) e^{\lambda^{*} \tau}\right] .
\end{aligned}
$$

Let $\lambda(\zeta, i)$ satisfy $\lambda(\zeta, i)-\lambda_{1}(\zeta, i)+\lambda_{2}(\zeta, i) e^{\lambda(\zeta, i) \tau}=0$. According to the definition of $\lambda^{*}$, it follows that

$$
\begin{aligned}
-\lambda^{*} & +\lambda_{1}(\zeta, i)-\lambda_{2}(\zeta, i) e^{\lambda^{*} \tau} \\
& =\left[-\lambda^{*}+\lambda_{1}(\zeta, i)-\lambda_{2}(\zeta, i) e^{\lambda^{*} \tau}\right]+\left[\lambda(\zeta, i)-\lambda_{1}(\zeta, i)+\lambda_{2}(\zeta, i) e^{\lambda(\zeta, i) \tau}\right] \\
& =\left(\lambda(\zeta, i)-\lambda^{*}\right)+\lambda_{2}(\zeta, i)\left[e^{\lambda(\zeta, i) \tau}-e^{\lambda^{*} \tau}\right] \geq 0 .
\end{aligned}
$$


Therefore

$$
z^{\prime}(\zeta)=x^{\prime}(\zeta)-y^{\prime}(\zeta)>k \bar{y}(0, r(0)) e^{-\lambda^{*} \zeta}\left[-\lambda^{*}+\lambda_{1}(\zeta, i)-\lambda_{2}(\zeta, i) e^{\lambda^{*} \tau}\right] \geq 0
$$

Obviously, this contradicts (3.7) and hence

$$
y(t, i) \leq \bar{y}(0, r(0)) e^{-\lambda^{*} t} \quad \text { for } t \in \mathbb{R}^{+} .
$$

Remark Obviously, if taking $\mathbb{S}=\{1\}$, then Proposition 1 reduces to [15], Lemma 2. So Proposition 1 extends and improves the corresponding result given in [15], Lemma 2.

Theorem 1 Let $(\mathrm{H} 1)$ and $(\mathrm{H} 2)$ hold. Assume that there exists a function $V \in C^{2,1}\left(\mathbb{R}^{n} \times\right.$ $\mathbb{R}^{+} \times \mathbb{S} ; \mathbb{R}^{+}$) such that

$$
c_{1}|x|^{p} \leq V(x, t, i) \leq c_{2}|x|^{p}
$$

and

$$
\mathscr{L} V(x, y, t, i) \leq-\lambda_{1}(t, i) V(x, t, i)+\lambda_{2}(t, i) V(y, t, i)
$$

for all $x, y \in \mathbb{R}^{n}, t \in \mathbb{R}^{+}$and $i \in \mathbb{S}$, where $p, c_{1}, c_{2}$ are positive numbers and $0<\lambda_{0} \leq \lambda_{1}(t, i)$, $0<\lambda_{2}(t, i) \leq q \lambda_{1}(t, i)$ with $0 \leq q<1$. Then

$$
\limsup _{t \rightarrow \infty} \frac{1}{t} \log \left(\mathbb{E}|x(t ; \xi)|^{p}\right) \leq-\lambda^{*}
$$

for all $\xi \in C_{\mathcal{F}_{0}}^{b}\left([-\tau, 0] ; \mathbb{R}^{n}\right)$, and

$$
\mathbb{E}\left(|x(t ; \xi)|^{p}\right) \leq \frac{c_{2}}{c_{1}} \mathbb{E}\left(\sup _{-\tau \leq s \leq 0}|\xi(s)|^{p}\right) e^{-\lambda^{*} t}
$$

where $\lambda^{*}>0$ is the unique root to the equation

$$
\lambda^{*}-\lambda_{1}(t, i)+\lambda_{2}(t, i) e^{\lambda^{*} \tau}=0 .
$$

In other words, the trivial solution of system (2.1) is pth moment exponentially stable and the pth moment Lyapunov exponent is not greater than $-\lambda^{*}$.

Proof For any small enough $\delta>0$, applying the generalized Itô formula, we have

$$
\begin{aligned}
\mathbb{E} V & (x(t+\delta), t+\delta, r(t+\delta))-\mathbb{E} V(x(t), t, r(t)) \\
& =\int_{t}^{t+\delta} \mathbb{E} \mathscr{L} V(x(s), x(s-\tau(s)), s, r(s)) d s \\
& \leq \int_{t}^{t+\delta}\left[-\lambda_{1}(s, r(s)) \mathbb{E} V(x(s), s, r(s))+\lambda_{2}(s, r(s)) \mathbb{E} V(x(s-\tau(s)), s, r(s))\right] d s \\
& \leq \int_{t}^{t+\delta}\left[-\lambda_{1}(s, r(s)) \mathbb{E} V(x(s), s, r(s))+\lambda_{2}(s, r(s)) \sup _{s-\tau \leq \theta \leq s} \mathbb{E} V(x(\theta), \theta, r(\theta))\right] d s .
\end{aligned}
$$


Taking $y(t, r(t))=\mathbb{E} V(x(t), t, r(t))$, then from inequality (3.4) we obtain

$$
d^{+} y(t, i) \leq-\lambda_{1}(t, i) y(t, i)+\lambda_{2}(t, i) \bar{y}(t, i)
$$

for all $r(t)=i \in \mathbb{S}$. Hence, it follows from Proposition 1 that

$$
y(t, i) \leq \tilde{y}(t, i) e^{-\lambda^{*} t}
$$

where $\tilde{y}(t, i)=\sup _{-\tau \leq s \leq 0} y(s, r(s))$.

On the other hand, by inequality (3.8), we get

$$
\begin{aligned}
\tilde{y}(t, i) & =\sup _{-\tau \leq s \leq 0} y(s, r(s))=\sup _{-\tau \leq s \leq 0} \mathbb{E} V(x(s), s, r(s)) \\
& \leq c_{2} \sup _{-\tau \leq s \leq 0} \mathbb{E}\left(|\xi(s)|^{p}\right) \leq c_{2} \mathbb{E}\left(\sup _{-\tau \leq s \leq 0}|\xi(s)|^{p}\right)
\end{aligned}
$$

and

$$
\mathbb{E}\left(|x(t)|^{p}\right) \leq \frac{1}{c_{1}} y(t, i)
$$

Hence

$$
\mathbb{E}\left(|x(t ; \xi)|^{p}\right) \leq \frac{c_{2}}{c_{1}} \mathbb{E}\left(\sup _{-\tau \leq s \leq 0}|\xi(s)|^{p}\right) e^{-\lambda^{*} t}
$$

where $\lambda^{*}$ is the unique positive root of the following equation:

$$
\lambda^{*}=\lambda_{1}(t, i)-\lambda_{2}(t, i) e^{\lambda^{*} \tau}, \quad i \in \mathbb{S} .
$$

Therefore, by Definition 1, we see that system (2.1) is $p$ th moment exponentially stable.

\section{Numerical examples}

In this section, we will discuss two examples to demonstrate the effectiveness of the obtained theory.

Example 1 Consider a one-dimensional stochastic differential equation with Markovian switching and time-varying delay of the form

$$
d(x(t))=f(x(t), t, r(t)) d t+g(x(t-\tau(t)), t, r(t)) d \omega(t)
$$

on $t \geq 0$, where $f, g: \mathbb{R} \times \mathbb{R}^{+} \times \mathbb{S} \rightarrow \mathbb{R}, \omega(t)$ is a scalar Brownian motion, and $r(t)$ is a right-continuous irreducible homogeneous Markov chain taking values in $\mathbb{S}=\{1,2\}$ with generator

$$
\Gamma=\left(\gamma_{i j}\right)_{2 \times 2}=\left[\begin{array}{cc}
-1 & 1 \\
1 & -1
\end{array}\right] .
$$


Assume that $\omega(t)$ and $r(t)$ are mutually independent, and

$$
x f(x, t, 1) \leq \frac{x^{2}}{4}, \quad x f(x, t, 2) \leq-6 x^{2}
$$

for all $(x, t) \in \mathbb{R} \times \mathbb{R}^{+}$, while

$$
|g(y, t, 1)| \leq \frac{|y|}{6}, \quad|g(y, t, 2)| \leq \frac{|y|}{7}
$$

for all $(y, t) \in \mathbb{R} \times \mathbb{R}^{+}$. To examine third moment exponential stability, we construct a Lyapunov function $V: \mathbb{R} \times \mathbb{R}^{+} \times \mathbb{S} \rightarrow \mathbb{R}^{+}$by

$$
V(x, t, i)=\alpha_{i}|x|^{3}
$$

with $\alpha_{1}=\alpha>0$ and $\alpha_{2}=1$. It is simple to show that the operator $\mathscr{L} V(x, y, t, i)$ from $\mathbb{R} \times$ $\mathbb{R} \times \mathbb{R}^{+} \times \mathbb{S}$ to $\mathbb{R}$ has the following form:

$$
\mathscr{L} V(x, y, t, i)=3 \alpha_{i}|x| x f(x, t, i)+3 \alpha_{i}|x||g(y, t, i)|^{2}+\left(\gamma_{i 1} \alpha+\gamma_{i 2}\right)|x|^{3} .
$$

By conditions (4.2) and (4.3), we have

$$
\mathscr{L} V(x, y, t, i)= \begin{cases}-(0.25 \alpha-1)|x|^{3}+0.0833 \alpha|x||y|^{2}, & \text { if } i=1, \\ -(19-\alpha)|x|^{3}+0.0612|x||y|^{2}, & \text { if } i=2 .\end{cases}
$$

Noting that

$$
|x||y|^{2} \leq \frac{1}{3}|x|^{3}+\frac{2}{3}|y|^{3},
$$

we obtain

$$
\mathscr{L} V(x, y, t, 1) \leq-(0.2222 \alpha-1)|x|^{3}+0.0556 \alpha|y|^{3}
$$

and

$$
\mathscr{L} V(x, y, t, 2) \leq-(18.9796-\alpha)|x|^{3}+0.0408 \alpha|y|^{3} .
$$

Choosing $\alpha$ as the solution to

$$
0.2222 \alpha-1=18.9796-\alpha
$$

that is, $\alpha=16.3472$, then we have

$$
\mathscr{L} V(x, y, t, i)= \begin{cases}-2.6324|x|^{3}+0.9089|y|^{3}, & \text { if } i=1, \\ -2.6324|x|^{3}+0.0408|y|^{3}, & \text { if } i=2 .\end{cases}
$$

Hence

$$
\mathscr{L} V(x, y, t, i) \leq-2.6324|x|^{3}+0.9089|y|^{3} .
$$


By Theorem 1, we conclude that the trivial solution of (4.1) is third moment exponentially stable.

Example 2 Consider the following one-dimensional stochastic differential equation with Markovian switching and time-varying delay:

$$
d x(t)=f(x(t), x(t-\tau(t)), r(t)) d t+g(x(t-\tau(t)), r(t)) d \omega(t),
$$

where $r(t)$ is a right-continuous Markov chain taking values in $\mathbb{S}=\{1,2\}$ with generator

$$
\Gamma=\left(\gamma_{i j}\right)_{2 \times 2}=\left[\begin{array}{cc}
-2 & 2 \\
1 & -1
\end{array}\right],
$$

$f(x(t), x(t-\tau(t)), 1)=-(3-0.5 \sin t) x(t)+2 x(t-\tau(t)), f(x(t), x(t-\tau(t)), 2)=-(2.25-$ $0.5 \sin t) x(t)+0.5 x(t-\tau(t)), g(x(t-\tau(t)), 1)=0.5 x(t-\tau(t)), g(x(t-\tau(t)), 2)=1.5 x(t-\tau(t))$, $\tau(t)=0.1|\cos t|, \omega(t)$ is a scalar Brownian motion. In this example, let $p=3, c_{1}=c_{2}=1$, $V(x, t, i)=x^{3}$. By a simple computation, we can easily get

$$
\begin{aligned}
\mathscr{L} V & (x(t), x(t-\tau(t)), 1) \\
= & 3|x(t)|^{2}[-(3-0.5 \sin t) x(t)+2 x(t-0.1|\cos t|)] \\
& +0.75|x(t)||x(t-0.1|\cos t|)|^{2}+\left(\gamma_{11}+\gamma_{12}\right)|x|^{3} \\
\leq & -(7.4167-1.5 \sin t)|x(t)|^{3}+1.1667|x(t-0.1|\cos t|)|^{3}
\end{aligned}
$$

and

$$
\begin{aligned}
\mathscr{L} V & (x(t), x(t-\tau(t)), 2) \\
= & 3|x(t)|^{2}[-(2.25-0.5 \sin t) x(t)+0.5 x(t-0.1|\cos t|)] \\
& +6.75|x(t)||x(t-0.1|\cos t|)|^{2}+\left(\gamma_{21}+\gamma_{22}\right)|x|^{3} \\
\leq & -(3.5-1.5 \sin t)|x(t)|^{3}+5|x(t-0.1|\cos t|)|^{3} .
\end{aligned}
$$

Hence, we have

$$
\mathscr{L} V(x(t), x(t-\tau(t)), r(t)) \leq-(3.5-1.5 \sin t)|x(t)|^{3}+5|x(t-0.1|\cos t|)|^{3} .
$$

By Theorem 1, we conclude that system (4.8) is the third moment exponentially stable. Numerical simulation further confirms the obtained results. Figures 1 and 2 show that the states $x$ of system (4.8) asymptotically approach zero indeed.

\section{Conclusions}

In this paper, we have investigated $p$ th moment exponential stability of stochastic differential equations with Markovian switching and time-varying delay. By using the Lyapunov function, the generalized Halanay inequality, and stochastic analysis techniques, some sufficient conditions are derived to ensure $p$ th moment exponential stability. The results obtained in this paper generalize and improve many well-known results. It is worth pointing 
Figure 1 Trajectory of $x(t)$ of system (4.8).

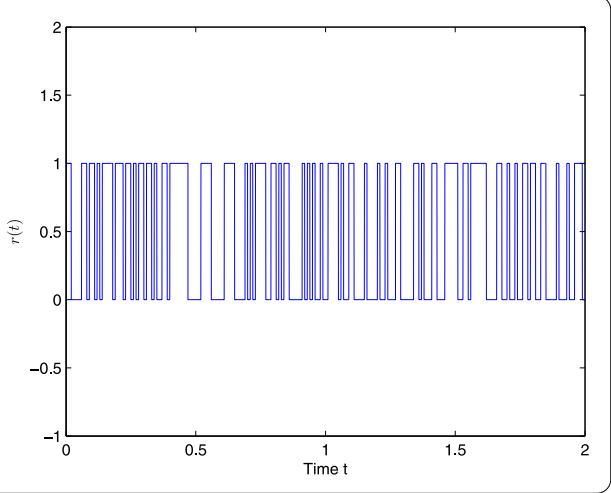

Figure 2 Trajectory of $r(t)$ of system (4.8).

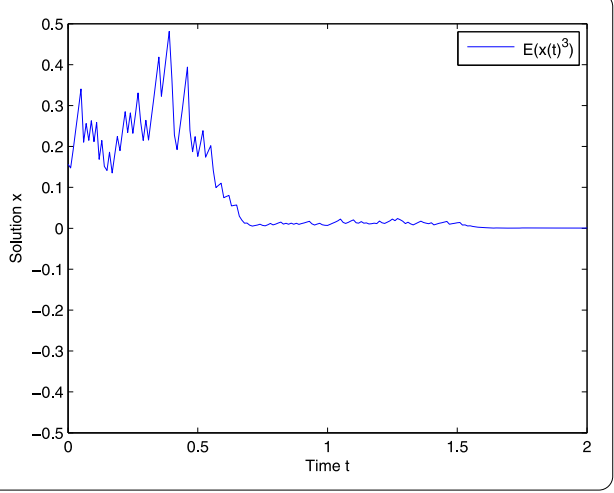

out that the contribution of this paper is that the traditional assumptions on the differentiability of the time-varying delay and the boundedness of its derivative are necessary in the earlier works. However, we take these restrictiveness away in this paper. Moreover, we present two examples to illustrate well the obtained results.

Competing interests

The authors declare that they have no competing interests.

\section{Authors' contributions}

The authors contributed equally and significantly in writing this article. The authors read and approved the final manuscript.

\section{Acknowledgements}

This work was supported in part by the National Natural Science Foundation of China under Grants nos. 11271087 , 11101434, Hunan Provincial Natural Science Foundation of China under Grant no. 12JJ4005, the Scientific Research Funds of Hunan Provincial Science and Technology Department of China under Grants no. 2013FJ4035, Humanities and Social Sciences Foundation of Ministry of Education of China under Grants no. 12YJAZH173, Changsha University of Science and Technology Innovation Foundation for Postgraduate no. CX2014YB19, and the Scientific Research Funds of Hunan Provincial Education Department of China under Grant no. 13C1036.

Received: 13 September 2014 Accepted: 1 April 2015 Published online: 17 April 2015

\section{References}

1. Arnold, L: Stochastic Differential Equations: Theory and Applications. Wiley, New York (1972)

2. Friedman, A: Stochastic Differential Equations and Their Application, vol. 2. Academic Press, New York (1976)

3. Has'minskii, RZ: Stochastic Stability of Differential Equations. Sijthoff \& Noordhoff, Moscow (1981)

4. Mohammed, SEA: Stochastic Functional Differential Equations. Longman, Harlow (1986)

5. Kolmanovskii, VB, Myshkis, A: Applied Theory of Functional Differential Equations. Kluwer Academic, Boston (1992)

6. Mao, X: Exponential Stability of Stochastic Differential Equations. Dekker, New York (1994)

7. Ji, Y, Chizeck, HJ: Controllability, stabilizability and continuous-time Markovian jump linear quadratic control. IEEE Trans. Autom. Control 35, 777-788 (1990)

8. Mariton, M: Jump Linear Systems in Automatic Control. Dekker, New York (1990)

9. Basak, GK, Bisi, A, Ghosh, MK: Stability of a random diffusion with linear drift. J. Math. Anal. Appl. 202, 604-622 (1996) 
10. Mao, X: Stability of stochastic differential equations with Markovian switching. Stoch. Process. Appl. 79, $45-67$ (1999)

11. Shaikhet, L: Stability of stochastic hereditary systems with Markov switching. Theory Stoch. Process. 2(18), 180-184 (1996)

12. Mao, X, Yuan, C: Stochastic Differential Equations with Markovian Switching. Imperial College Press, London (2006)

13. Huang, C, He, Y, Huang, L, Zhu, W: pth moment stability analysis of stochastic recurrent neural networks with time-varying delays. Inf. Sci. 178(9), 2194-2203 (2008)

14. Zhu, E, Yuan, Q: pth moment exponential stability of stochastic recurrent neural networks with Markovian switching. Neural Process. Lett. 38, 487-500 (2013)

15. Cao, J, Wang, J: Absolute exponential stability of recurrent neural networks with time-varying delays. Neural Netw. 17(3), 379-390 (2004)

Submit your manuscript to a SpringerOpen ${ }^{\circ}$ journal and benefit from:

- Convenient online submission

Rigorous peer review

- Immediate publication on acceptance

- Open access: articles freely available online

- High visibility within the field

- Retaining the copyright to your article 